Leprosy

\section{Global leprosy elimination: time to change more than the elimination target date}

\section{N Durrheim, R Speare}

\section{Contentious elimination target requires rethinking}

D espite the World Health Assembly's enthusiastic adoption in 1991 of a resolution to "eliminate leprosy as a public health problem by the year 2000", it remains an important cause of global chronic neurological disability. ${ }^{1}$ The optimistic belief that leprosy could be conquered despite a limited understanding of its epidemiology was principally based on the availability of effective multidrug treatment (MDT), consisting of a combination of rifampicin, dapsone, and clofazimine. ${ }^{23}$

For elimination purposes, "public health problem" was defined as less than one case of leprosy per 10000 population the assumption being that below this prevalence level, loosely based on historical experience in Scandinavia and Western Europe, reduced transmission of Mycobacterium leprae would result in decreased incidence of infection and natural leprosy extinction. Disease prevalence was the measure chosen by the World Health Organisation (WHO) because of scepticism that incidence could be measured by routine surveillance systems due to leprosy's variable and comparatively long incubation period, the insidious onset of clinical disease, the tendency of many infected patients to self heal, and the chronic nature of disease. ${ }^{4}$

The WHO is proud of the "success" of the leprosy elimination campaign. More than 10 million patients have received MDT therapy; the number of registered patients has decreased from 5 million in 1985 to less than a million in 2001; of 122 countries considered endemic in 1985, 107 have achieved the elimination target at country level; and by the end of 2000 the global prevalence of leprosy was reported as below 1 case per 10000 population. ${ }^{5}$ These accomplishments provided the impetus for extending the deadline for achieving the elimination goal at country level to 2005 .

How confident can we be that WHO's optimism is justified?

The choice of prevalence as the measure for determining elimination is fundamentally flawed. The practice of reporting point prevalence at the end of a calendar year does not provide an accurate proxy of leprosy incidence or transmission, as it is dependent on the duration of infection, or in the case of the leprosy elimination campaign, the period that a patient remains on a treatment register. Thus, the cleaning of registers, removing patients that had died, been cured or been on treatment for indefinite prolonged periods, had a striking immediate effect on prevalence. However, the single greatest influence on prevalence was the WHO's decision to reduce the treatment period of lepromatous leprosy patients from 24 to 12 months. ${ }^{67}$ This literally halved the global burden of registered leprosy cases. The recent drive by the WHO to further reduce the treatment period to only six months for all leprosy patients irrespective of disease classification will certainly achieve global country elimination! $!^{8}$

The other aspect of concern in the leprosy elimination campaign is that countries are not required to provide evidence of the effectiveness of their surveillance for leprosy. The poliomyelitis elimination campaign and country targets for detection of cases of acute flaccid paralysis is a good example of how a proxy measure can ensure surveillance meets a desired standard. ${ }^{9}$ For leprosy, merely having a highly ineffective health service that failed to diagnose cases would result in the elimination target being attained.

During 2001, 719330 leprosy cases were detected globally. ${ }^{5}$ If the assumption that reduced prevalence equates to decreased transmission is correct, then we would expect that fewer new cases would be detected as prevalence reduces. However, this has not occurred and the converse is true, with an increase in the number of cases detected in many leprosy endemic countries. In the six countries that currently account for almost $90 \%$ of new leprosy registrations, incident cases rose between 1995 and 2000. ${ }^{10}$ This increase has been explained as merely reflecting increased detection of "backlog" hidden cases through improved outreach services and special campaigns. Although this is likely to be true in certain situations, particularly where patients with advanced disease or disability are detected, it does not adequately explain why children constitute $15 \%$ of new cases. ${ }^{5}$ The latter provides evidence of ongoing active transmission of $M$ leprae.

The focus on country as the epidemiological unit has political merit, but seems flawed as it ignores the tendency for leprosy to cluster at a level below the country level. Profound heterogeneity in leprosy detection after elimination suggests that choosing "country" as the resolution level for the elimination target is inappropriate. Evidence of extended nasal carriage of $M$ leprae DNA in lepromatous patients as well as transient excretion by asymptomatic people may partially explain the local clustering phenomenon. ${ }^{11}$ Perhaps, rather than a single measure of leprosy elimination at the country level, two measures should now be used; the standard < 1 per 10000 at the country level, and an additional measure, of the proportion of all health districts within the country that have attained the elimination level. A country, therefore could meet the country target, but still not meet elimination criteria if some districts had leprosy.

There are an increasing number of countries that have achieved the "elimination" level but new case detection continues unabated. Although it is not yet precisely clear what underpins ongoing transmission, indirect immunological evidence by skin testing is consistent with the hypothesis that transient subclinical human infection with a variable period of infectiousness facilitates transmission. ${ }^{12}{ }^{13}$ South Africa is a good example of a country where, since leprosy became notifiable in 1921, prevalence levels have remained well below the elimination target level but new cases continue to occur. ${ }^{14}$

If reduction in treatment duration will not assure "point prevalence" leprosy elimination, then the drive to integrate leprosy activities into general primary service provision is likely to achieve this goal! As medical conditions becomes increasingly rare, successful detection by health workers is impaired by lack of diagnostic experience and a decreased index of suspicion. ${ }^{15}$ The increasing prevalence of HIV associated dermatological conditions that may mimic leprosy is an additional challenge to early diagnosis in HIV endemic countries. The choice of a flawed elimination target focused at country level, use of point prevalence as the indicator of successful elimination, lack of measurable criteria to assure effective surveillance, and the concerted drive towards leprosy service integration and increasingly abbreviated treatment courses, should temper our interpretation of progress toward global leprosy elimination. However, it will not be simple to achieve a more accurate 
representation of the true situation. Until a steady epidemiological situation exists with no more "hidden" cases, leprosy detection rates will not accurately reflect incidence.

The lack of convincing evidence that leprosy transmission has been substantially reduced has already elicited calls to withdraw from elimination and to rather focus on patient management and rehabilitation. ${ }^{16}$

We cannot afford to further dilute the resources necessary for effective leprosy control. Available polymerase chain reaction technology should be more actively harnessed to assist our understanding of the epidemiology of this ancient disease, particularly the contributory role of subclinical infection to disease persistence in specific settings. ${ }^{17-19}$ A sustained effort is clearly merited in those countries that are still to achieve the dubious elimination level, but an energetic refocusing of efforts should occur in countries that have achieved "elimination" to identify endemic districts. Recent research in Mpumalanga Province, South Africa confirmed marked heterogeneity of leprosy occurrence "post-elimination" and suggested that within high risk districts all treated patients and their intimate contacts should be included in an active surveillance programme to ensure early diagnosis of subsequent cases and prevention of disability. ${ }^{14}$
Leprosy "elimination" may be imminent, but there is no room for complacency as achieving the WHO target will not safeguard us against a re-emergence of leprosy.

$J$ Epidemiol Community Health 2003;57:316-317

\section{Authors' affiliations}

D N Durrheim, R Speare, School of Public Health and Tropical Medicine, James Cook University, Townsville, Australia

Correspondence to: Professor D N Durrheim, School of Public Health and Tropical Medicine James Cook University, Townsville, 4811, Australia; David.Durrheim@jcu.edu.au

\section{REFERENCES}

1 World Health Assembly. Elimination of leprosy: resolution of the 44th World Health Assembly. Geneva: World Health Organization, 1991. (Resolution no WHA 44.9.)

2 World Health Organziation Expert Committee on Leprosy. Seventh report. World Health Organ Tech Rep Ser 1998;874:20.

3 Noorden SK. Epidemiology and control of leprosy-a review of progress over the last 30 years. Trans $R$ Soc Trop Med Hyg 1993:87:515-17.

4 Declercq E. Prevalence: a valid indicator for monitoring leprosy "elimination"? Int J Lepr Other Mycobact Dis 2001;69:1 1 1-13.

5 World Health Organization. Leprosy. Global situation. Wkly Epidemiol Rec 2002;77: 1-8.

6 World Health Organization. Shortening duration of treatment of multibacillary leprosy. Wkly Epidemiol Rec 1997;72:125-8.
7 Fine PE, Warndorff DK. Leprosy by the year 2000-what is being eliminated? Lepr Rev 1997;68:201-2

8 Lockwood DN. Leprosy elimination-a virtual phenomenon or a reality? BM 2002:324:1516-18.

9 Hinman AR, Foege WH, de Quadros CA, et al. The case for global eradication of poliomyelitis. Bull World Health Organ 1987;65:835-40.

10 World Health Organization. Leprosy. Wkly Epidemiol Rec 2001;76:173-9.

11 Klatser PR, van Beers S, Madjid B, et al. Detection of Mycobacterium leprae nasal carriers in populations for which leprosy is endemic. J Clin Microbiol 1993;31:2947-51.

12 Cree IA Smith WC. Leprosy transmission and mucosal immunity: towards eradication? Lepr Rev 1998;69:112-21.

13 Fine PEM. Leprosy: the epidemiology of a slow bacterium. Epidemiol Rev 1982:4:161-8

14 Durrheim DN, Fourie A, Balt E, et al. Leprosy in Mpumalanga Province, South Africa-Eliminated or hidden? Lepr Rev 2002;73:326-33.

15 Durrheim DN, Speare R. Measles elimination - a case definition to enhance surveillance. Commun Dis Intell 2000;24:329-31.

16 Visschedijk J, van de Broek J, Eggens $\mathrm{H}$, et al. Mycobacterium leprae-millennium resistant! Leprosy control on the threshold of a new era. Trop Med Int Health 2000;5:388-99.

17 Noorden SK. Leprosy research and elimination. Lepr Rev 2000;71:S12-14.

18 Colston MJ. Mycobacterium leprae genome sequence: a landmark achievement. Lepr Rev $2001 ; 72: 385-6$

19 Hatta M van Beers SM, Madiid B, et al. Spatial distribution and persistence of Mycobacterium leprae nasal carriage among a population in which leprosy is endemic in Indonesia. Trans $\mathrm{R}$ Soc Trop Med Hyg 1995;89:381-5 